\title{
Reactions at polymer interfaces: A Monte Carlo Simulation
}

\author{
M. Müller ${ }^{1,2}$ \\ ${ }^{1}$ Department of Physics, Box 351560, University of Washington \\ Seattle, Washington 98195-1560 \\ and \\ ${ }^{2}$ permanent address: Institut für Physik, Johannes Gutenberg Universität \\ D-55099 Mainz, Germany
}

February 28, 2018

\begin{abstract}
Reactions at a strongly segregated interface of a symmetric binary polymer blend are investigated via Monte Carlo simulations. End functionalized homopolymers of different species interact at the interface instantaneously and irreversibly to form diblock copolymers. The simulations, in the framework of the bond fluctuation model, determine the time dependence of the copolymer production in the initial and intermediate time regime for small reactant concentration $\rho_{0} R_{g}^{3}=0.163 \cdots 0.0406$. The results are compared to recent theories and simulation data of a simple reaction diffusion model. For the reactant concentration accessible in the simulation, no linear growth of the copolymer density is found in the initial regime, and a $\sqrt{t}$-law is observed in the intermediate stage.
\end{abstract}

\section{Introduction}

Reactions at interfaces have attracted great interest because of a variety of practical applications. They are involved in facilitated transport across biological membranes, and in selective removal of a component of a mixture by contact with a reactive liquid [1]. Recent interest stems from extensive commercial application of reactive polymer blending technique[2, 3] and progress of the experimental studies 4 , 5, 6. Melt blending of different polymer species (denoted A and B) is widely employed[7] to improve the application properties of polymeric materials. Since the entropy of mixing of high-molecular-weight polymers is extremely low, macromolecular alloys can often be described as an assembly of interfaces between relatively pure phases. Block copolymers containing both 
types of monomers can be used to tailor the properties of polymer-polymer interfaces. They promote mixing by means of reducing the interfacial tension and the droplet-droplet coalescence rate 4 ] and mechanically strengthening the composite material due to entanglements across the interface. An effective method of creating copolymers at the interface is in-situ production[2], e.g. chemical reactions of end functionalized homopolymers.

Recently, reactions at a flat interface in a binary, symmetric polymer blend have been studied independently and in some detail by O'Shaughnessy and Sawhney 8, 9 and Fredrickson [10 and Milner 11]: A and B polymers are assumed to be immiscible, but structurally symmetric. The almost pure phases $\mathrm{A}$ and $\mathrm{B}$ host a small fraction $\rho_{0}$ of chains with a terminal reactive group. The $\mathrm{A}$ reactive ends can only react with $\mathrm{B}$ reactive ends and form $\mathrm{AB}$ diblockcopolymers at the interface between the bulk phases. The reactions are instantaneous and irreversible. The interfacial density $\sigma$ of copolymers at the interface grows according to the rate equation:

$$
\frac{d \sigma}{d t}=K(t) \rho_{0}^{2}
$$

where $t$ measures the duration of the reaction and $\rho_{0}$ denotes the time independent fraction of reactive chains in the bulk. The reaction rate $K$ is largely independent from the details of the microscopic mechanism, because subdiffusive monomer motion on small time scales guarantees reaction whenever the coils of two reactive chains overlap 12. For small number density $\rho_{0}$ of reactive chains $\left(\rho_{0} R_{g}^{3} \ll 1\right.$ with $R_{g}$ radius of gyration) and high incompatibility, Fredrickson and Milner 11 predict three time regimes: At times smaller than $\tau=D / K_{0}^{2} \rho_{0}^{2}$, the density of reactive chains at the interface remains close to its bulk value. The reaction rate within the framework of the Rouse model [13] is time-independent, and given by [8, 10]: $K_{0} \approx 100.6 D R_{g}^{2} / \ln N$. It follows that the interfacial density of copolymers grows linearly with time in this regime. At times larger than $\tau[14$, there is a depletion hole of reactive chains with spatial extension $\sqrt{D t}$, and the reaction rate is determined by the flux of reactive chains to the interface. The reaction rate decays as $K=\sqrt{D / \pi \rho_{0}^{2} t}$, i.e. the interface acts much like an absorbing boundary. The copolymer density grows like $t^{1 / 2}$ in this regime. For even longer times $t>\Phi^{2} R_{g}^{2} / N^{2} D \rho_{0}^{2}$ ( $\Phi$ monomer density), the copolymer accumulates at the interface and forms a brush. This prevents reactive chains from reaching the interface, and the reaction rate becomes extremely small, decaying as $1 / t \sqrt{\ln t}$. The copolymer density increases only as $\sqrt{\ln t}$.

In spite of the practical applications of interfacial reactions, qualitative experiments to determine the time dependence of the copolymer concentration at the interface are still rare 2, 3, 4, 15. Their interpretation is complicated by structural asymmetries between the two homopolymer species, or the reactive and inert polymers of the same species. Furthermore, large differences in the mobilities of the species lead to qualitative modifications of the reaction 
kinetics [16]. In such a situation, Monte Carlo simulations can provide valuable information for understanding the reactions in these inhomogeneous, complex fluids and for exploring the validity of approximations in the analytic treatment. Such simulations of reactions at surfaces and of catalysis of small molecules have proven extremely useful in studying the stochastic dynamics of reaction-diffusion processes 17. The present simulations are the first to investigate reactions at polymer interfaces, and they cover the initial and intermediate time regime. They yield the time evolution, as well as the spatial depletion profile, of reactants at the interface. We compare our results to the theories described above and to a simple reaction-diffusion model.

\section{Model and simulation technique}

If the microscopic reaction is sufficiently fast, the reaction rate is largely independent of the microscopic mechanisms [8, 12]. Since the study of reaction kinetics poses high computational demands, we employ a coarse grained and highly computational efficient lattice model of a dense polymeric melt. The Bond Fluctuation Model (BFM) 18] retains the relevant features of polymeric materials: connectivity of the monomers along a chain molecule, excluded volume interaction of the monomeric units, and a short range thermal interaction potential. The BFM has proven very useful for studying various related properties of dense polymeric melts. Among them are single chain dynamics in athermal melts 19, 20] and binary blends 21, living polymerization 22], adsorption in polymer brushes [23], and interfacial properties in binary 24, 25] and ternary blends 26]. In the framework of the BFM each segment occupies all eight sites at the corners of a unit cell in a simple cubic lattice, and no site can be doubly occupied. Adjacent monomers along a chain molecule are connected via one of 108 bond vectors. These are chosen such that the local selfavoidance prevents chains from crossing each other during their motion. The fluctuating bond length permits the implementation of random, local monomer displacements 18 such that the dynamics of an isolated chain is well describable by the Rouse model[19, 20]. We work at a filling fraction $8 \Phi=0.5$ of occupied lattice sites, and all length are measured in units of the lattice spacing $u$. At this density the BFM reproduces many characteristics of a dense polymer melt. The blend consists of two species of homopolymers, denoted A and B, of equal chain length $N=32$, and diblock copolymers AB of chain length 64 . This is well below the chain length $N=200$, where first signs of repation-like motion are observed. We employ a square well potential, which encompasses the first peak of the pair correlation function, i.e. comprising all 54 neighbor sites up to a distance $\sqrt{6}$ lattice units: $\epsilon=\epsilon_{A B}=-\epsilon_{A A}=-\epsilon_{B B}=0.1 k_{B} T$. Monomers of the same kind attract each other, whereas there is a repulsion between unlike species. This incompatibility corresponds to $\chi N \approx 17$, and is well inside the strong segregation limit for binary blends. Both, the interfacial properties of 
the binary blend 24, 25] as well as the equilibrium behavior of copolymers at interfaces [26, 27 have been investigated extensively.

We work in a $64 \times 64 \times 128$ geometry, and for the highest fraction of reactive chains additional data were obtained for system size $64 \times 64 \times 256$. Periodic boundary conditions in all three spatial directions were applied, such that the systems contain two interfaces parallel to the xy-plane. The initial configurations for the binary blend have been carefully equilibrated using $10^{6}$ local MCS. (On the average each monomer attempts to jump once within a Monte Carlo Step/MCS). After this initial equilibration of the interfacial properties, the equilibration was continued and starting configurations for the reaction study were collected every 50,000 MCS. For the smaller system size we average our results over 96 independent simulation runs, corresponding to a total of more than 3 million monomers. For the larger size we employ 30 independent configurations. Simulations were performed on a CONVEX SPP1200, using a trivial parallelization strategy.

Some interfacial properties of the starting configurations are presented in Fig. 1. The blend is well segregated, i.e. the coexisting phases consist almost entirely of $\mathrm{A}$ or $\mathrm{B}$ polymers. Between the coexisting phases there is an interface of width $w \approx 3.7$. The conformational properties have been discussed in detail in Ref. 24, 25. We simply emphasize that, in agreement with mean field theories 25, there is a pronounced segregation of chain ends at the interface and a concomitant depletion at a distance $R_{g}$. The total monomer density is somewhat reduced at the center of the interface.

In these equilibrated starting configurations a fraction of homopolymers was randomly chosen to bear a reactive end, and the reaction was started at time $t=0$. This corresponds to an experimental situation in which a (fast diffusive) reactive agent/catalyst[2] is added to the blend, or the reaction is initiated by radiation. O'Shaughnessy and Sawhney [8, 9] analyzed the qualitative dependence on the monomeric reaction probability. For very low monomeric reaction probability $Q$, correlation in the density of reactive particles are only weakly perturbed by the reactions, and the reaction rate $K$ is proportional to the equilibrium contact probability of reactive ends. However, this mean field kinetics is only appropriate if the reaction probability is smaller than a crossover value $Q^{\star} \sim 1 / \ln N[8,9]$. Therefore, reactions in high molecular weight polymers are typically diffusion controlled. This diffusion controlled reaction kinetics is largely independent from the details of the monomeric reaction mechanism, especially from the monomeric capture radius and reaction probabilities. Since the simulation can treat only rather short polymers, we use a large monomeric reaction rate $Q$ and capture radius to make sure that we work in the controlled regime. If the distance between reactive $\mathrm{A}$-ends and $\mathrm{B}$-ends is less than the capture radius $\sqrt{6}$, reactive homopolymers form $\mathrm{AB}$ diblock copolymers instantaneously and irreversibly. Of course, the concrete value of the capture radius $\sqrt{6}$ has no physical significance; it is the largest value the model allows to be compatible with local monomer dynamics and chain connectivity. According to 
analytical treatments [8, 9, 10, 11], we expect our results to be characteristic for diffusion controlled kinetics and rather independent of the concrete monomeric reaction mechanism (e.g. the model also applies to long polymers with a large but finite monomeric reaction rate).

Otherwise the reactive chains behave the same as the non-reactive polymers. We considered systems with three different values of the initial number density of reactive chains in their corresponding bulk phases: $\rho_{0}=1 / 2048,1 / 4096$, and $1 / 8192$. With the measured radius of gyration $R_{g}=6.93$, the scaled number density ranged in our simulation from $\rho_{0} R_{g}^{3}=0.041$ to 0.1625 . Furthermore, we determined the self-diffusion constant from the mean square displacements in the plane parallel to the interface for the largest system size to $D=1.17(5)$. $10^{-4} u^{2} / \mathrm{MCS}$, which is very close to the corresponding athermal value.

\section{$3 \quad$ Simulation results}

Upon activating the reactive polymers, $\mathrm{AB}$ diblock copolymers are created at the interface. The time dependence of the interfacial density for different fraction of reactive chains is presented in Fig. 2. For the highest fraction, the simulation data for the two system sizes agree to within the statistical accuracy. Since finite size effects are expected to be even smaller for lower fractions of reactive chains, they can be neglected for our data. In contrast to the predictions above, the simulation data for the copolymer interfacial density do not exhibit a linear growth law at small times. Due to the high initial reaction rate in the Monte Carlo simulation, the theory underestimates the interfacial density over the entire time covered by the simulation for the two largest reactant concentrations. The broken lines in the figure show the theoretical prediction for the diffusive growth in the intermediate time regime. For the lowest density, the simulation data are rather well described by the prediction for the intermediate regime. However the agreement is fortuitous, as the simulation data cover mainly the initial time regime $\left(\tau \approx 3 \cdot 10^{5} \mathrm{MCS}\right)$.

To eliminate the influence of the fast initial rate, it is instructive to monitor the growth rate $K(t)$. The solid lines in Fig. 2 2 show fits to the time development of the copolymer density which are used to determine the reaction rate. These rates are presented in Fig. 3 in scaled form. The solid lines show the simulational results, while the dashed ones correspond to the theoretical predictions in the initial $(t<\tau)$ and intermediate $(t>\tau)$ regimes. First order corrections 11 to describe the crossover between the two regimes are also included into the figure:

$$
\begin{array}{llll}
\frac{d \sigma / d t}{K_{0} \rho_{o}^{2}}=1-\frac{4}{\sqrt{\pi}}\left(\frac{t}{\tau}\right)^{1 / 2} & \text { for } & t \ll \tau & \text { initial regime } \\
\frac{d \sigma / d t}{K_{0} \rho_{o}^{2}}=\frac{1}{\sqrt{\pi}}\left(\frac{t}{\tau}\right)^{-1 / 2}-0.254\left(\frac{t}{\tau}\right)^{-3 / 4} & \text { for } & t \gg \tau & \text { intermediate regi(ß3 })
\end{array}
$$


Even for $\rho_{0} R_{g}^{3}$ as small as 0.0406 the prediction underestimates the reaction rate by an order of magnitude, and there is no sign of a constant reaction rate at small times. Furthermore the simulation data for different fractions of reactive chains do not collapse onto a single curve. In the intermediate time regime, however, the simulation data are consistent with the theoretical prediction $K \sim 1 / \sqrt{t}$, and the anticipated proportionality constant is in rough agreement with the simulation data, though the asymptotic limit $t / \tau \gg 1$ is not reached for the lower fractions of reactive chains.

In Fig. 1 we present the non-equilibrium profiles across the interface for $\rho_{o} R_{g}^{3}=0.163$ and $t / \tau \approx 24.4$. The copolymers accumulate at the interface, and their composition profile resembles its equilibrium shape, as determined in Ref. [27. The copolymers do not yet form a brush at the interface. Therefore the simulation data are still in the intermediate regime. The homopolymer density profile, however, is altered as they are displaced from the interface. The simulation data also confirm the predicted depletion of reactive chains in the interfacial zone, and the growth of this depletion hole with time. Thus the simulation data corroborate the main features of the theory in the intermediate time regime, but disagree strongly with predictions in the initial stage.

There are several possible reasons for the difference in the initial stage between the simulation data and the theory:

The local equilibrium structure of the homopolymer interface exhibits an enrichment of chain ends at the interface. Therefore the initial reaction rate is expected to be higher than that for a uniform initial distribution of reactive segments. This effect has been used to rationalize the enhanced reaction rate in PS-PMMA interfaces 15. However, it is not clear to what extent this correlation in the initial condition accounts quantitatively for the discrepancies between simulation and theory. Futhermore, the theory deals with Rouse chains at an interface in the Helfand regime $1 \gg \chi \gg 1 / N$. This latter condition can be satisfied only for very long chains, whereas the chain length $N$ must not be too large so that the chain dynamics is describable by the Rouse model.

Additionally, the reactant density $\rho_{o} R_{g}^{3}=0.0406$ in the simulations might still not be small enough to meet the condition $\rho_{o} R_{g}^{3} \ll 1$ assumed in the theory. The constant reaction rate should be observable for times much larger than the relaxation time $\tau_{R}$ of the internal chain motion, but much smaller than the crossover time $\tau$ to the intermediate regime. Note however, that the crossover time scale $\tau$ and the Rouse time $\tau_{R}=6 R_{g}^{2} / \pi^{2} D \approx \tau\left(78.6 \rho_{0} R_{g}^{3} / \ln N\right)^{2}$ are not well separated for the reactant concentration studied. Unfortunately, simulation at even lower fraction of reactive chains pose very high statistical demands, which are beyond our computational facilities. 


\section{Reaction diffusion model}

We decided to compare our results to additional simulations of a simple random walk model. Bulk reactions $[12$ in polymeric systems can be modeled schematically by random walkers which react if their mutual distance is smaller than the radius of gyration in the corresponding polymer system. Therefore we consider two types of random walkers, denoted $\mathrm{A}$ and $\mathrm{B}$, on a simple cubic lattice. A-type walkers are restricted to the half-space $z \geq 0$, whereas B-type walkers are confined to $z \leq 0$. An A-type walker annihilates with a B-type walker (and vice versa), whenever their mutual distance is smaller than $R$. The initial time regime for a class of similar models has been discussed by Durning and O'Shaugnessy [16]. The model is an appropriate caricature of polymer reactions at interfaces for the initial and intermediate time regime. Since it eliminates the shortest time scales, which are associated with the segmental motion on distances smaller than the radius of gyration, and also neglects the inert homopolymer matrix, much lower volume fractions for reactive particles are accessible in the simulations. Furthermore, many of the above-mentioned complications characteristic of polymeric systems are absent. We work with a capture radius $R=8$ and a $256 \times 256 \times 512$ geometry, and a $1024 \times 1024 \times 2048$ system for the lowest density. Initially, the random walkers are uniformly distributed in their volumes. We employ at least 512 independent systems.

The reaction rate in its scaled form as well as the prediction of Fredrickson and Milner 11 are presented in Fig. 5. For very small density of walkers $\rho_{0} R^{3}<0.002$, the simulations show an almost constant initial reaction rate, in agreement with the predictions. The crossover to the intermediate regime, and the diffusive growth in the intermediate regime, is described by the theory in the limit of vanishing reactant concentration, and the corresponding scaling function is obtained as the lower envelope of the simulation data. The spatial density profile (cf. Fig. 5 inset) in the intermediate time regime exhibits a pronounced depletion hole, and is qualitatively similar to the profile obtained in the simulation of the polymer system. In the vicinity of the interface there are some deviations due to the finite interfacial width in the polymer system.

For concentrations comparable to those used in the simulation of the polymer systems, the reaction diffusion model shows similar deviations, i.e. a decaying reaction rate in the initial stage which is always larger than in the limit $\rho_{0} \rightarrow 0$. However the deviations are somewhat smaller than for the polymer system. This is partially due to the fact, that the reaction in the polymer system does not take place instantaneous when the chain volumes of the reactive pair overlap, but the reaction is retarded by a time of the order $\tau_{R}$. Therefore we conclude that the finite concentration of reactive chains accounts qualitatively for the discrepancies between the analytic theory and simulations of the polymeric system. However, the simple reaction-diffusion model cannot describe the polymeric system quantitatively for finite reactant concentrations. 


\section{Conclusions and outlook}

In summary, we have presented extensive simulations of reactions at interfaces, both for polymeric systems as well as for a simple reaction-diffusion model. We have compared our simulation results to recent theoretical predictions [8, 9, 10, 11. At intermediate times we find agreement between the theory and the simulations of both models. The initial state and the crossover to the intermediate regime is described by the theory only for extremely small concentration of reactive particles. These are not accessible in the simulation of the polymeric systems, but are in the simulation of the random walker model. Results of the latter are compatible with the theoretical predictions in the limit $\rho_{0} R_{g}^{3} \rightarrow 0$, and the simulations yield estimates for the complete crossover scaling behavior of the reaction rate. However, for non-zero but small concentrations of reactive particles, the reaction rate is grossly underestimated by the theory. Even for the smallest fraction of reactive chains simulated in the present study, $\rho_{0} R_{g}^{3}=0.0406$, the initial reaction rate obtained from the simulation is about an order of magnitude larger than predicted, and is not constant, but decays with time. It should be emphasized that our results are not an artifact of the small chain length used in the simulation. The reactant concentrations correspond to weight fractions of $25 \%, 12.5 \%$ and $6.25 \%$. These weight fractions are within the experimentally relevant range. If one increases the chain length at

fixed weight fraction, then $\rho_{0} R_{g}^{3}$ increases as $\sqrt{N}$ taking the initial condition even further from the assumptions of the theory. Therefore we expect that experiments will also observe a decay of the reaction rate over the entire time regime, unless extremely small volume fractions of reactive chains are used.

Further studies might investigate the crossover between the diffusive growth and the late regime, in which the copolymer brush hinders the reactive chains to reach the surface. This regime is accessible for high concentration of reactive chains. At high incompatibilities the copolymers cannot reduce the surface tension completely, and there is a first order transition to a lamellar phase at high copolymer concentration 26. The direct measurement of the interactions between copolymer "coated" interfaces 26 is an interesting topic, because it relates to the important effect of a reduced droplet-droplet coalescence rate. In the weak segregation limit, however, the addition of copolymer causes a continuous transition to the disordered phase to be encountered, (the Scott-line which exists for $2<\chi N<3$ within mean field theory [28]). Thus it would be interesting to study the temperature dependence of the reaction kinetics at weak and intermediate segregations, where reactions might lead to complete miscibility.

\section{Acknowledgement}

It is a great pleasure to thank M. Schick for valuable discussions and critical reading of the manuscript. Financial support by the Alexander von Humboldt 
foundation and the National Science Foundation under Grant No. DMR9531161 as well as a generous allocation of computer time on the CONVEX SPP 1200 of the computing center in Mainz, Germany, are gratefully acknowledged.

\section{References}

[1] Astarita, G Mass Transfer with Chemical Reactions, Elsevier, New York 1967.

[2] Stewart, M.E.; George, S.E.; Miller, R.L.; Paul, D.R. Polym.Eng and Sci 1993, 33,675 .

[3] Ide, F.; Hasegawa, A. J.Appl.Poly.Sci. 1974, 18, 963.

[4] Sundararaj, U.; Macosko, C.W. Macromolecules 1995, 28, 2647.

[5] C.E. Scott, and C.W. Macosko, Polymer 35, 5442 (1994).

[6] A description of recent experimental progress can be found in Macromolecular Symposia: Polymer Blends 112 141-175 (1996).

[7] Solc, C. (edt.) Polymer Compatibility and Incompatibility, Principles and Practices, Haarwood Academic, Chur, 1980.

Han, C.D. (edt.) Polymer Blends and Composites in Multiphase Systems, ACS, Washington D.C..

Cahn, R.W.; Haasen, P.; Kramer, E.J.; Materials Science and Technology, A Comprehensive Treatment, Vol 12, VCH, Weinheim 1993.

[8] O'Shaughnessy, B.; Sawhey, U. Phys.Rev.Lett. 1996, 76, 3444.

[9] O'Shaughnessy, B.; Sawhey, U. Macromolecules 1996, 29, 7230.

[10] Fredrickson, G.H. Phys.Rev.Lett. 1996, 76, 3440.

[11] Fredrickson, G.H.; Milner, S.T. Macromolecules 1996, 29, 7386.

[12] Doi, M. Chem.Phys. 1975, 9, 455.

Doi, M. Chem.Phys. 1975, 11, 107 and 115.

deGennes, P.G. 1982, J.Chem.Phys. 76, 3316.

[13] Rouse, P.E. J.Chem.Phys. 1953, 21, 1272.

[14] The crossover time $\tau$ can be estimated by equating the number of copolymers produced per area in the initial regime (i.e. for times smaller than $\tau) K_{0} \rho_{0}^{2} \tau$ and the reduction of copolymers per area in the depletion hole $\rho_{0} \sqrt{D \tau}$ at time $\tau$. 
[15] Guegan, P.; Macosko, C.W.; Ishizone, T.; Nakahama, S. Macromolecules 1994, 27, 4993.

[16] Durning, C.; O’Shaughnessy, B. J.Chem.Phys. 1988, 88, 7117.

[17] see e.g. Gonzalez, A.P.; Pereyra, V.D.; Milchev, A.; Zgrablich, G. Phys.Rev.Lett. 1995, 75, 3955.

Satulovsky, J; Albano, E.V.; J.Chem.Phys. 1992, 97, 9441.

[18] Carmesin, I.; Kremer, K. Macromolecules 1988, 21, 2819.

Deutsch, H.P.; Binder, K. J.Chem.Phys. 1991, 94, 2294.

[19] Paul, W.; Binder, K.; Heermann, D.; Kremer, K. J.Chem.Phys. 1991, 95, 7726.

[20] Müller, M.; Wittmer, J.P.; Cates, M.E. Phys.Rev.E 1996, 53, 5063.

[21] Müller, M.; Binder, K.; J.Phys. II France 1996, 6, 187.

[22] Rouault, Y.; Milchev, A. Phys.Rev.E 1995, 51, 5903.

[23] Kopf, A.; Baschnagel, J.; Wittmer, J; Binder, K Macromolecules 1996, 29, 1433.

[24] Müller, M.; Binder, K.; Oed, W. Faraday Trans. 1995, 91, 2369.

[25] Schmid, F.; Müller, M. Macromolecules 1995, 28, 8639.

[26] Müller, M.; Schick, M. J.Chem.Phys. 1996, 105, 8885.

[27] Werner, A.; Schmid, F.; Binder, K.; Müller, M. Macromolecules 1996, 29, 8246.

[28] Broseta, D.; Fredrickson, G.H. J.Chem.Phys. 1990, 93, 2927. 


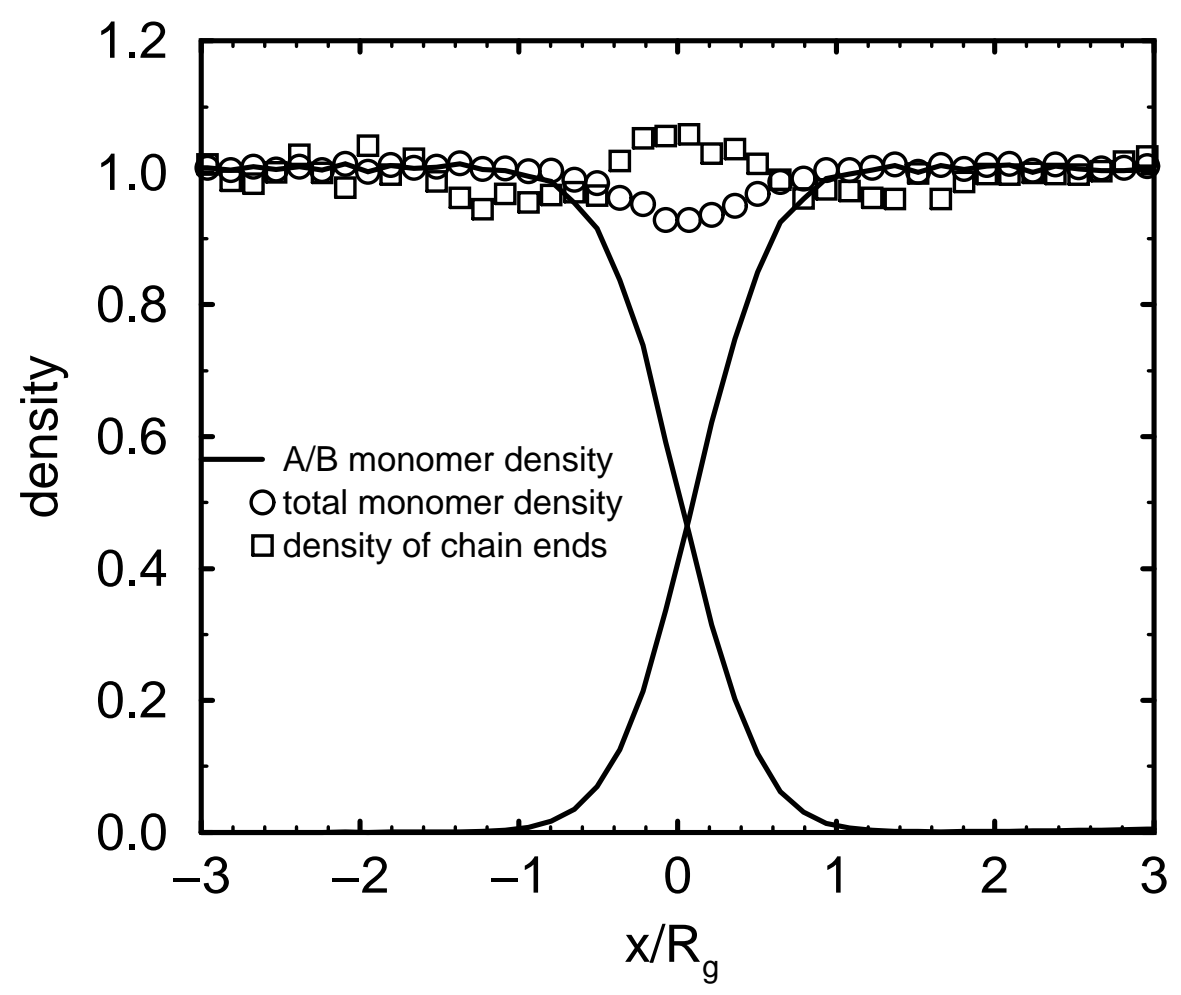

Figure 1: Interfacial properties of the equilibrated starting configuration: The solid lines present the normed density of A and B monomers, wheras the symbols denote the total monomer density (circles) and the density of chain ends (squares) scaled by a factor $N / 2$. Note the enrichment of chain ends at the center of the interface. 


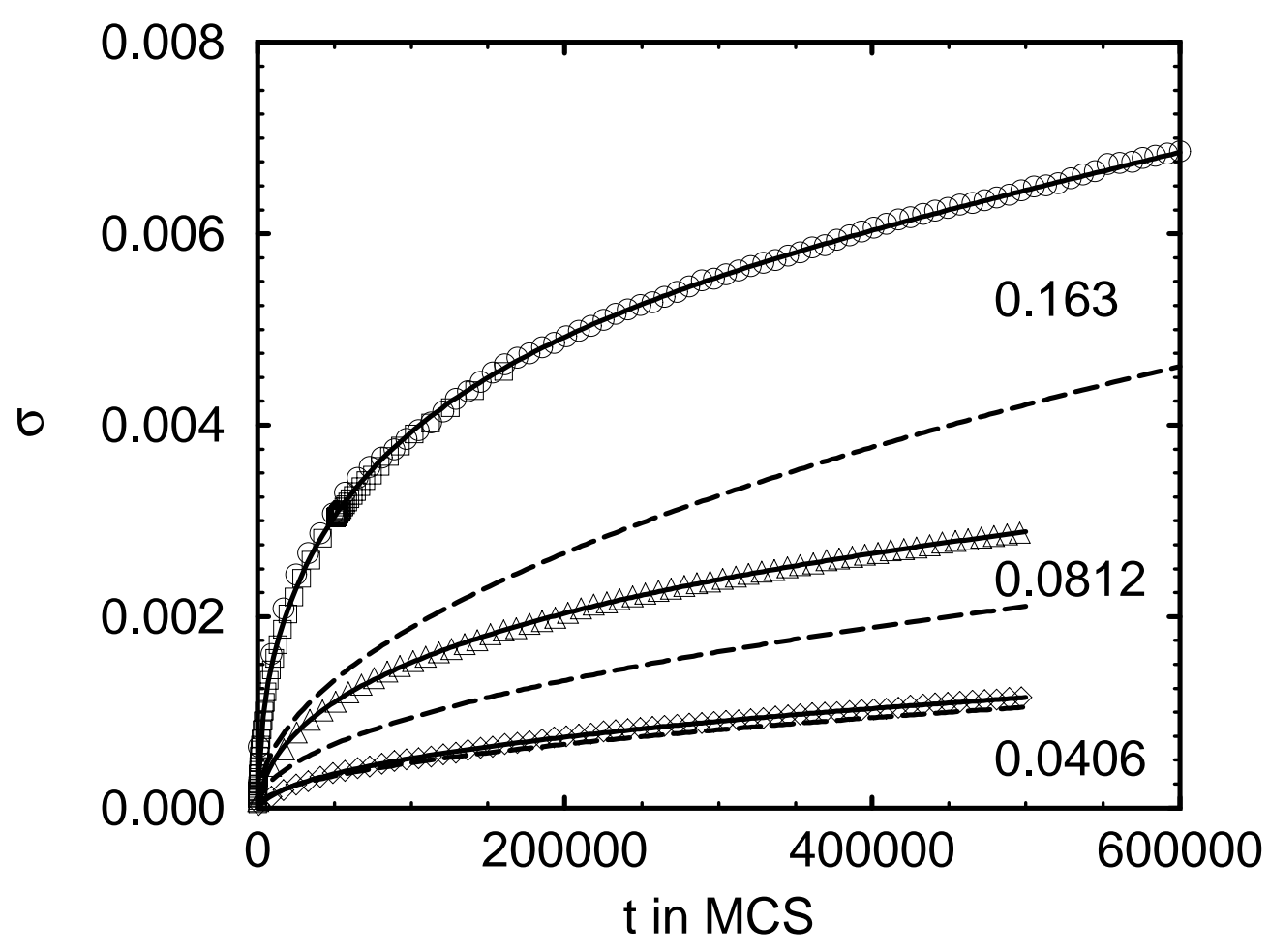

Figure 2: Time dependence of the copolymer concentration at the interface: circles denote simulation data for $\rho_{0}=1 / 2048$ and $64 \times 64 \times 256$ system size, squares correspond to $\rho_{0}=1 / 2048$, triangles to $\rho_{0}=1 / 4096$, diamonds to $\rho_{0}=1 / 8192$ and system size $64 \times 64 \times 128$ respectively. The solid lines are fits to the simulation raw data, whereas the dashed lines represent the predicted time development in the intermediate regime. 


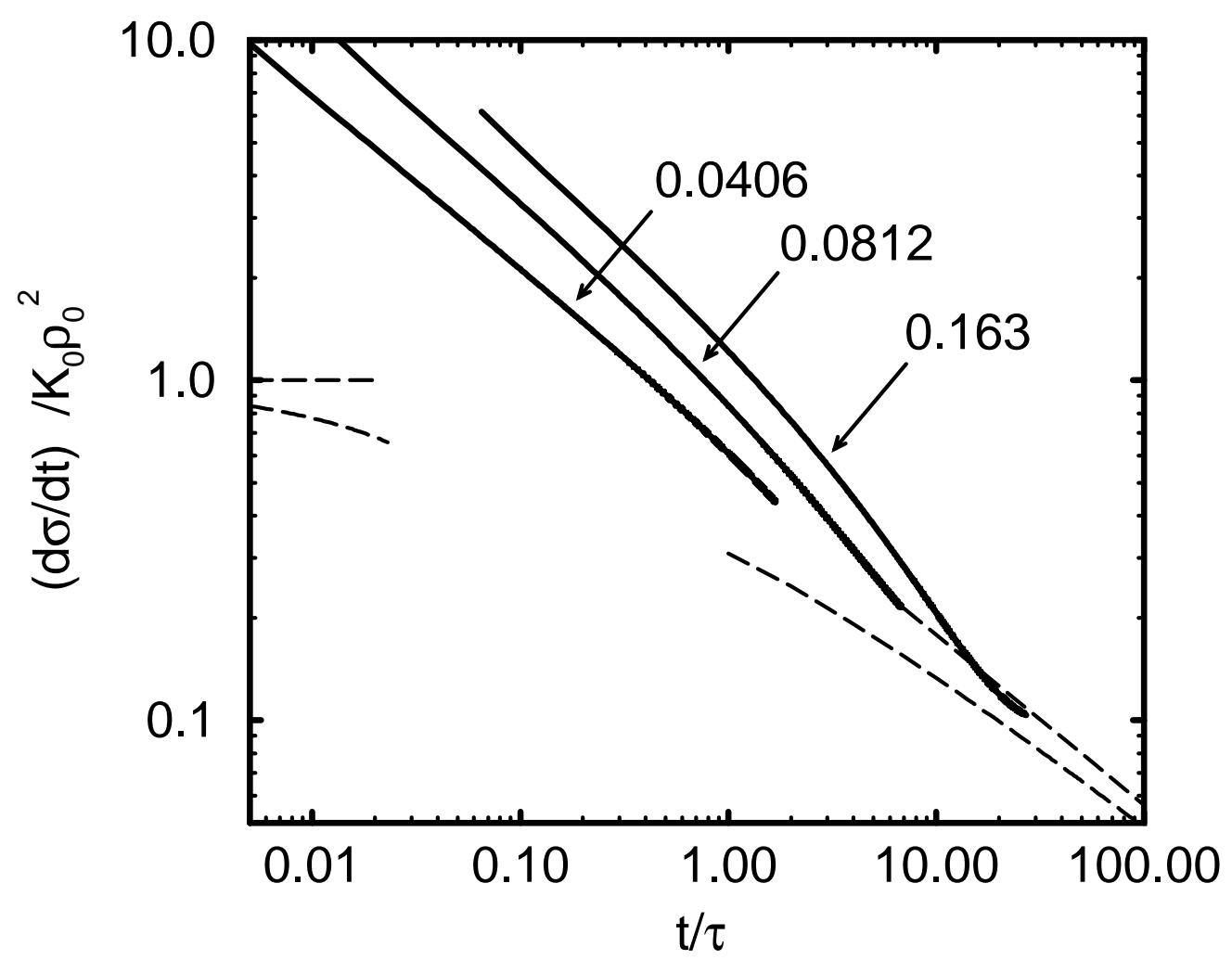

Figure 3: Scaled reaction rate $\frac{d \sigma / d t}{K_{0} \rho_{0}^{2}}$ for the polymer system: The solid lines are the results of the Monte Carlo simulation for different values $\rho_{0} R_{g}^{3}$ as indicated in the figure. The two set of dashed lines correspond to the predictions of Fredrickson and Milner[11] for $\rho_{0} R_{g}^{3} \ll 1$. The higher values correspond to the asymptotic results for small and large values of $t / \tau$; the lower ones present first order corrections. 


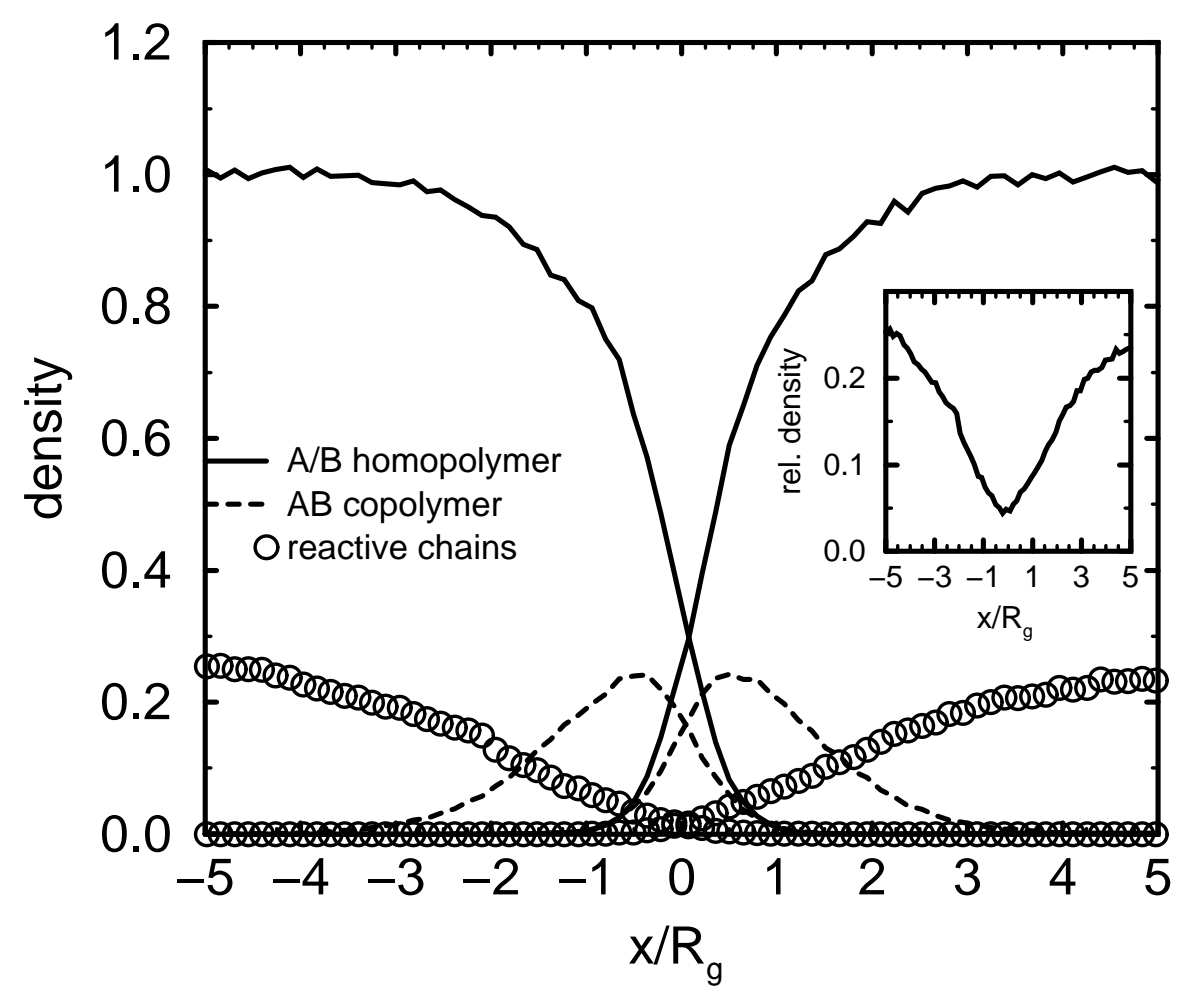

Figure 4: Non-equilibrium density profiles for $\rho_{0} R_{g}^{3}=0.163$ and $t / \tau=24.4$. The solid lines present the monomer density of the A and B segments of the homopolymers. The dashed lines correspond to the A and $\mathrm{B}$ monomers of the copolymers, whereas the monomer density of the two types of reactive chains is denoted by circles. The inset shows the ratio of the monomer density of reactive chains to the total monomer density of homopolymers. 


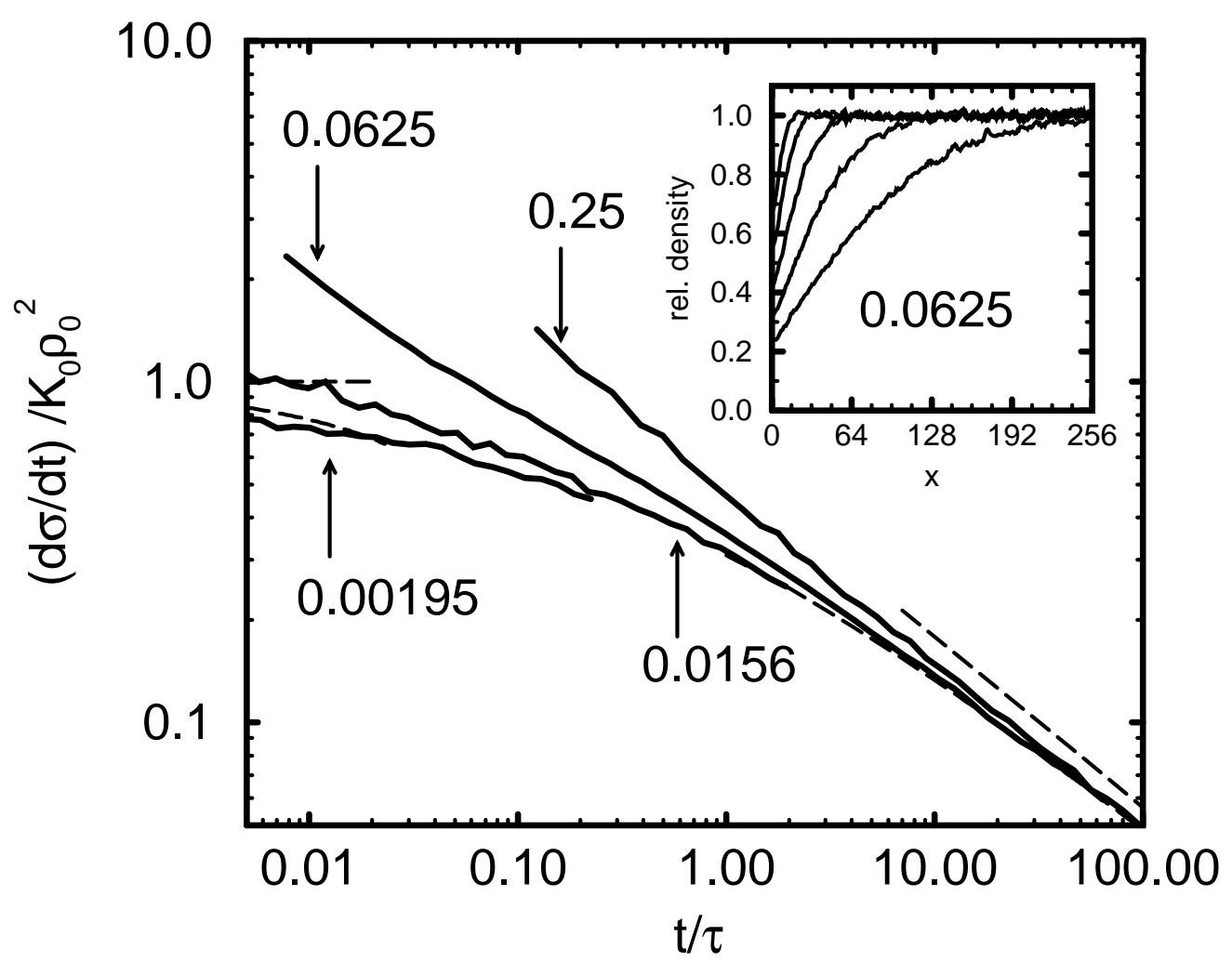

Figure 5: Scaled reaction rate for the reaction diffusion system: The solid lines are the result of the Monte Carlo simulations, the values of $\rho_{0} R_{g}^{3}$ being indicated in the figure. The dashed lines are the same as in Fig.3. Only for very small concentration does one observe a reaction rate which is in agreement with the predictions [9]. The initial reaction rate is $K_{0} \approx 157$

The inset presents the density profiles of reactants for $\rho_{0} R_{g}^{3}=0.0625$ and $t / \tau=0.28,1.13,4.53,18.12$ and 72.49 . 